FEDSM2002-31026

\title{
A REDUCED-ORDER MODEL OF HEAT TRANSFER EFFECTS ON THE DYNAMICS OF BUBBLES
}

\author{
Al Preston \\ Tim Colonius* \\ Christopher E. Brennen \\ Division of Engineering and Applied Science \\ California Institute of Technology \\ Pasadena, CA 91125 \\ email: colonius@caltech.edu
}

\begin{abstract}
The Rayleigh-Plesset equation has been used extensively to model spherical bubble dynamics, yet it has been shown that it cannot correctly capture damping effects due to mass and thermal diffusion. Radial diffusion equations may be solved for a single bubble, but these are too computationally expensive to implement into a continuum model for bubbly cavitating flows since the diffusion equations must be solved at each position in the flow. The goal of the present research is to derive reduced-order models that account for thermal and mass diffusion. We present a model that can capture the damping effects of the diffusion processes in two ODE's, and gives better results than previous models.
\end{abstract}

\section{INTRODUCTION}

A continuum model that couples the Rayleigh-Plesset equation for bubble dynamics with the equations of continuity and momentum (van Wijngaarden 1968, 1972) has been used extensively in the computation of bubbly cavitating flows. Recent examples include Shimada et al. (1999),Wang (1999), Colonius et al. (2000) and Preston et al. (2002). A significant limitation is the use of a polytropic approximation to account for the expansion and compression of the gas bubble interior and an effective liquid viscosity to account for damping of the bubble radial

*Address all correspondence to this author. motion due to heat transfer (Prosperetti et al. 1988, Kameda \& Matsumoto 1996). The correct treatment of the thermal effects requires the solution of the radial energy equation in each bubble and the surrounding liquid; however this is an expensive computation.

Previous models that account for thermal diffusive effects include the models of Prosperetti (1991) valid near either the isothermal or adiabatic limits. These models work well in the limits for which they were intended, but are not accurate for behavior between the two limits. Storey \& Szeri (2001) developed a model that switches between isothermal and adiabatic behavior depending upon relative timescales. While this approach yielded good estimates of peak bubble temperatures during bubble collapse, it is unable to correctly capture attenuation of bubble rebounds due to thermal damping effects. Lertnuwat et al. (2001) proposed a model that estimated the thermal energy flux out of the bubble by using an average bubble temperature and an estimation of the thermal penetration length. This seems a reasonable approach near the adiabatic limit, but is clearly not reasonable when the thermal penetration length approaches or even exceeds the bubble radius.

We propose an alternative thermal model that is able to capture thermal damping effects over a wide range of applications. The thermal model requires only one additional ODE to be integrated alongside the Rayleigh-Plesset equation. The accuracy of the thermal model is tested by comparing the model response 
of a single forced bubble to a computation in which the full energy equation in the bubble interior is solved. Results show that the proposed thermal model produces closer agreement to full computations than previous models, particularly cases where the amount of attenuation of bubble rebounds is important.

Finally, we present an extension of the thermal model that incorporates mass diffusion of vapor in the bubble. Preliminary results indicate that the mass transfer model is also able to capture the extent of the initial expansion and the attenuation of the bubble rebounds very well. The mass transfer model requires one additional ODE to be integrated.

\section{THERMAL MODEL}

The thermal model is based on the simplified set of equations of Prosperetti et al. (1988) for a gas bubble with the internal pressure assumed to be spatially uniform. This assumption enables the derivation of the following ordinary differential equation for the internal bubble pressure,

$$
\frac{d p}{d t}=\frac{3 \gamma}{R}\left(\left.\frac{p_{o} D}{R} \frac{\partial T}{\partial y}\right|_{y=1}-p \frac{d R}{d t}\right),
$$

which is coupled to the Rayleigh-Plesset equation ${ }^{1}$ for the motion of the liquid,

$$
R \frac{d^{2} R}{d t^{2}}+\frac{3}{2}\left(\frac{d R}{d t}\right)^{2}+\frac{4}{R e R} \frac{d R}{d t}+\frac{2}{W e R}=p-p_{\infty}(t) .
$$

The variables in the above equations have been nondimensionalized as $R=R^{\prime} / R_{o}^{\prime}, T=T^{\prime} / T_{o}^{\prime}, p=p^{\prime} / \rho_{L}^{\prime} R_{o}^{\prime 2} \omega_{o}^{\prime 2}$, while $y=r^{\prime} / R^{\prime}(t)$ is the radial coordinate chosen to fix the bubble wall at $y=1$. The dimensionless gas diffusivity, Reynolds number and Weber number are given respectively as $D=$ $k^{\prime} / \rho_{o}^{\prime} c_{p}^{\prime} R_{o}^{\prime 2} \omega_{o}^{\prime}, R e=R_{o}^{\prime 2} \omega_{o}^{\prime} / v_{L}^{\prime}$ and $W e=\rho_{L}^{\prime} R_{o}^{\prime 3} \omega_{o}^{\prime 2} / S^{\prime}$, where $\omega_{o}^{\prime}$ is the bubble natural frequency. The non-dimensional initial internal bubble pressure is computed from equilibrium of Eq. (2) as, $p_{o}=p_{\infty_{o}}+2 / W e$, where $p_{\infty_{o}}$ is the non-dimensional ambient pressure.

The ordinary differential Eqs. (1) and (2) are typically closed by the radial energy equation for the temperature distribution in the bubble, that is coupled directly to Eq. (1) through the temperature gradient at the bubble wall. We focus on ways to estimate the temperature gradient at the bubble wall without solving the energy equation. From linear analysis of the energy equation in the frequency domain, we can write,

$$
\left.\frac{\partial \hat{T}^{\prime}}{\partial y}\right|_{y=1}(\omega)=-\Psi(\omega) \hat{\bar{T}}^{\prime}(\omega),
$$

where the transfer function $\Psi(\omega)$ is,

\footnotetext{
${ }^{1}$ The thermal model can readily be used with other forms of the RayleighPlesset equation that include effects of liquid compressibility.
}
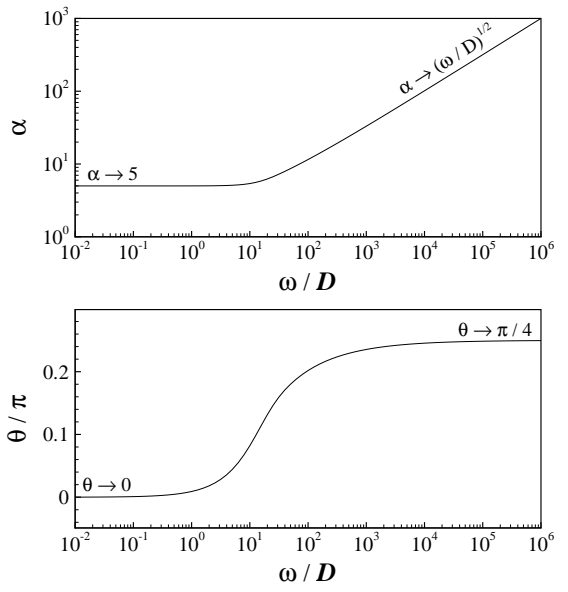

Figure 1. Magnitude, $\alpha$, and phase, $\theta$ of the transfer function, $\Psi=$ $\alpha e^{i \theta}$, versus $\omega / D$.

$$
\Psi(\omega)=\left\{\left[\sqrt{\frac{i \omega}{D}} \operatorname{coth} \sqrt{\frac{i \omega}{D}}-1\right]^{-1}-3 \frac{D}{i \omega}\right\}^{-1} .
$$

Here primes denote small fluctuations, $\hat{T}(\omega)$ is the Fourier transform of $T(t)$, and the overbar denotes a quantity averaged over the bubble volume. The magnitude, $\alpha$, and phase, $\theta$, of $\Psi=\alpha e^{i \theta}$ are plotted as functions of frequency in Fig. 1. We see in the low frequency, or nearly isothermal, limit $\alpha \rightarrow 5$ and $\theta \rightarrow 0$ so that Eq. (3) may be inverted exactly. Away from the isothermal limit we avoid the convolution integral resulting from the inversion by making the crude approximation of setting $\Psi(\omega)=\Psi\left(\omega_{c}\right)$ in Eq. (3), where $\omega_{c}$ is a characteristic frequency of bubble motion. This allows the inversion to be written as,

$$
\left.\frac{\partial T^{\prime}}{\partial y}\right|_{y=1}(t)=-\alpha\left(\omega_{c}\right) \bar{T}^{\prime}(t+\Delta t),
$$

where $\Delta t=\theta\left(\omega_{c}\right) / \omega_{c}$, represents a phase difference between the temperature gradient at the bubble wall and the average bubble temperature. The next approximation that we make is to neglect the phase difference and extend the model to the non-linear regime. The final approximation is written as,

$$
\left.\frac{\partial T}{\partial y}\right|_{y=1}(t) \approx-\alpha\left(\omega_{c}\right)[\bar{T}(t)-1] .
$$

Finally, we use the perfect gas law to approximate the volume averaged bubble temperature and density,

$$
\begin{aligned}
\bar{T} & \approx p / \bar{\rho}, \\
\Rightarrow \bar{T} & \approx p R^{3},
\end{aligned}
$$

where we have also used the assumption that the pressure within the bubble is spatially uniform. Equations (1) and (2) together 
with the approximations (6) and (8) constitute the final form of the thermal model. It can be shown that the thermal model is identical to the full non-linear equations in the nearly isothermal limit, while away from this limit the use of $\alpha\left(\omega_{c}\right)$, computed from Eq. (4), gives the best agreement of the linearized forms of the thermal model and full equations.

While it is clear that for harmonically forced bubbles the characterisitic frequency, $\omega_{c}$, should be taken as the driving frequency, it is not so clear what value of $\omega_{c}$ should be used in more general applications. Therefore in all computations we use the linear natural bubble frequency for setting the model parameter, which under the present non-dimensionalization is achieved by setting $\omega_{c}=1$. Although this approximation is crude, our results demonstrate that it works well in a wide range of computations.

\section{RESULTS}

\section{Harmonic Forcing}

The thermal model is intended for application to general forcings that may arise in continuum bubbly model flows, such as the nozzle flow of Preston et al. (2002). Rather than test the model with specific examples we use harmonic forcings over a wide range of frequencies and amplitues. The harmonic forcing field is given by,

$$
p_{\infty}(t)=p_{\infty_{o}}\left(1+A \sin \omega_{f} t\right),
$$

where $A$ is the non-dimensional amplitude and $\omega_{f}$ is the forcing frequency non-dimensionalized by the linear natural bubble frequency.

Fig. 2 shows a bifurcation diagram of the computed bubble radius sampled at every period of the forcing, for a $10 \mu \mathrm{m}$ radius bubble driven at a forcing frequency $\omega_{f}=0.8$ with an incrementally increasing driving pressure amplitude. The curve for the thermal model is almost identical to the full computation ${ }^{2}$ through the first and second subharmonic bifurcations at $A \approx 1.61$ and 1.90 . At $A \approx 1.96$ the full computation and thermal model both predict chaotic behavior for which the exact form was found to be very sensitive to tolerances in the numerical integration as well as the rate at which the driving pressure amplitude was increased.

We also compare the thermal model with the polytropic model (using the effective values of polytropic index, $k_{\text {eff }}$, and damping ratio, $\beta \equiv \mu_{e f f}^{\prime} / \mu_{L}^{\prime}$, obtained to match linear theory (Prosperetti et al. 1988)). The curve for the polytropic model with effective damping $(\beta=6.35)$, while maintaining the same general form as the full computation, is shifted significantly to the right and slightly below the full computation. The polytropic model without effective damping $(\beta=1)$ yields results that agree

\footnotetext{
${ }^{2}$ The full computation solves the energy equation for the bubble interior using a Chebychev spectral collocation method with an adaptable number of modes (Kamath \& Prosperetti 1989, Hao \& Prosperetti 1999).
}

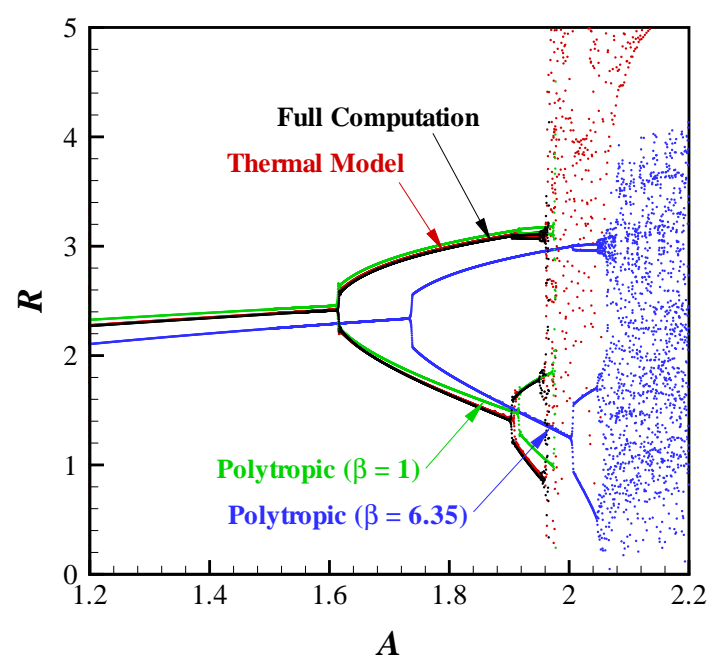

Figure 2. Bifurcation diagram of the bubble radius sampled at every period of the forcing pressure for an air bubble of equilibrium radius $R_{0}^{\prime}=10 \mu m$ in water driven with forcing frequency $\omega_{f}=0.8$ and a slowly increasing pressure amplitude, $A$. Ambient conditions are $20^{\circ} \mathrm{C}$ and 1 atmosphere. The curves show comparisons to the full computation of the thermal model and the polytropic model with and without effective damping $(\beta=6.35$ and 1 respectively). The effective polytropic index for the polytropic model is $k_{\text {eff }}=1.079$ and parameters for the thermal model are $D=0.108$ and $\alpha=5.184$.

much more closely with the full computation. It appears that the addition of effective damping to the polytropic model substantially delays the onset of the bifurcations as well slightly overdamps the bubble response.

Figure 3 shows the response of a $10 \mu \mathrm{m}$ bubble forced with non-dimensional pressure amplitude $A=0.6$ over a range of frequencies. This graph plots (for a given forcing frequency, $\omega_{f}$ ) the maximum value of bubble radius attained during a steady oscillation. The thermal model shows excellent agreement to the full computation over all range of frequencies, even though the model was tuned for forcing at the bubble natural frequency. By contrast, the polytropic model is unable to correctly predict the location and magnitude of the harmonic peaks, and the behavior of the model depends strongly on the (arbitrary) value of effective damping that is used.

We also constructed bifurcation diagrams and frequency response curves for a $50 \mu \mathrm{m}$ bubble, which showed similar trends as the $10 \mu \mathrm{m}$ bubble. For both bubble sizes the thermal model gives results that agree with the full computation much more closely than the polytropic model with or without effective damping.

\section{Gaussian Forcing}

While the thermal model shows excellent agreement to the full computation for harmonic forcings of a range of frequencies 


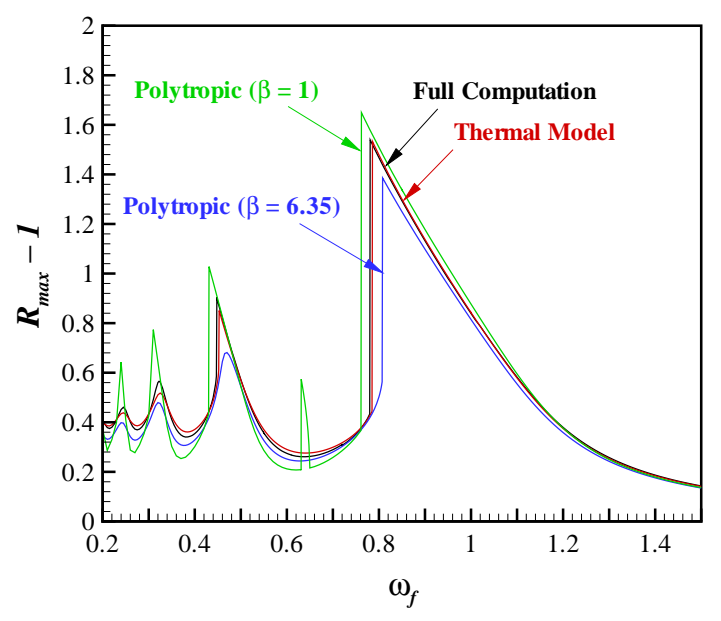

Figure 3. Frequency-response curves for the forced oscillations of an air bubble of radius $R_{0}^{\prime}=10 \mu \mathrm{m}$ in water for a dimensionless pressure amplitude $A=0.6$. Ambient conditions are $20^{\circ} \mathrm{C}$ and 1 atmosphere. The curves show comparisons to the full computation of the thermal model and the polytropic model with and without effective damping $(\beta=6.35$ and 1 respectively). The effective polytropic index for the polytropic model is $k_{\text {eff }}=1.079$ and parameters for the thermal model are $D=0.108$ and $\alpha=5.184$.

and amplitude, we now wish to test the model for more complex forcings. Single bubbles are subjected to the following Gaussian decrease in far field pressure,

$$
p_{\infty}(t)=p_{\infty_{o}}\left(1-A \exp \left[-\left(\left(t-t_{o}\right) / t_{w}\right)^{2}\right]\right),
$$

which has been chosen to approximately represent the pressure that would be experienced by a bubble that is convected through the nozzle of Preston et al. (2002). Figures 4 and 5 compare computed bubble radii of different models to the full computation for initial equilibrium radii of 4 and $40 \mu m$, respectively. For the small $4 \mu m$ bubble (Fig. 4) the behavior is very close to the isothermal limit, and as anticipated the thermal model curve is essentially identical to the full computation. In addition the nearly isothermal model of Prosperetti agrees almost exactly with the full computation, since this is within the limit for which the model is valid. The switching model of Storey \& Szeri (2001) underestimates the attenuation because there is no thermal damping in either the isothermal or adiabatic limits. The polytropic model with effective damping underpredicts the initial growth, probably due to too much viscous damping that results from using an effective viscosity.

For the large 40 $\mathrm{mm}$ bubble the behavior departs significantly from the isothermal limit. Figure 5 shows that the thermal model captures the initial bubble growth and attenuation of bubble rebounds reasonably well, but the periods of the rebounds are slightly overpredicted. The absence of thermal damping in the

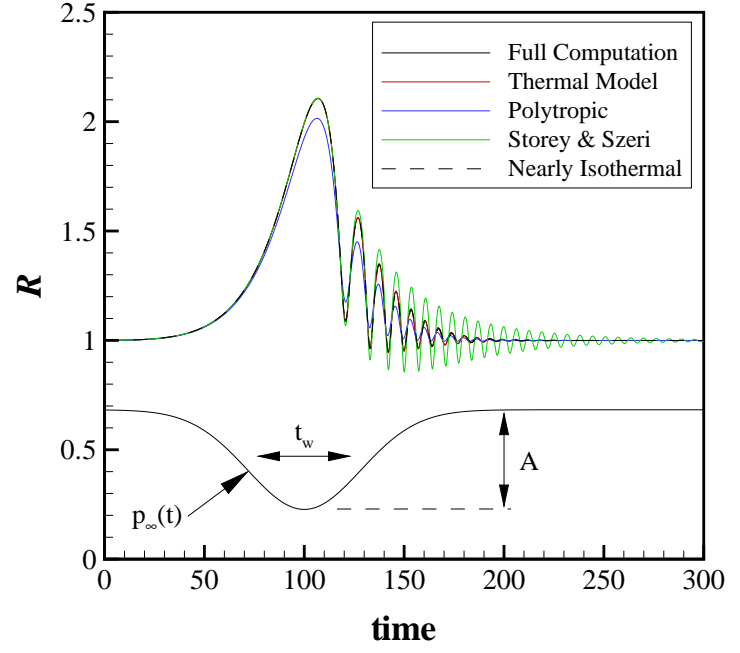

Figure 4. Computed bubble radius for an air bubble of equilibrium radius $R_{0}^{\prime}=4 \mu m(D=0.217)$ in water subject to a Gaussian decrease in far field pressure $\left(A=1.0, t_{w}=40.0\right)$. Ambient conditions are $20^{\circ} \mathrm{C}$ and 1 atmosphere. The curves show comparisons to the full computation of the thermal model $(\alpha=5.089)$, the polytropic model with effective damping ( $\beta=2.64, k_{\text {eff }}=1.027$ ), the switching model of Storey \& Szeri (2001), and the nearly isothermal model of Prosperetti et al. (1991).

switching model of Storey \& Szeri (2001) is clearly shown by the slow decay of the rebounds. By contrast, the nearly isothermal model of Prosperetti severely overpredicts the attenuation of the bubble rebounds. The polytropic model also behaves poorly, since the use of a polytropic index of $k_{\text {eff }}=1.213$ prevents the correct prediction of the initial expansion which is relatively slow and therefore nearer the isothermal limit. It seems that the present thermal model can correctly capture this nearly isothermal expansion even though we use a value of $\alpha$ that has been chosen to match behavior away from this limit.

In Fig. 6 we present plots of the temperature gradient at the bubble wall as a function of average bubble temperature for the computations in Figs. 4 and 5. The present model represents this relation as a straight line with slope $\alpha$ (cf. Eq. (6)). The behavior for the full computation can be remarkably different. For the small $4 \mu \mathrm{m}$ bubble (nearly isothermal behavior) there is a small hysterisis loop in the full computation, although the loop is very thin and always close to the single line of the thermal model. For the large $40 \mu \mathrm{m}$ bubble the hysterisis effect is far more pronounced and it is evident that the thermal model severely underestimates the temperature gradient at the point around the minimum radius (maximum average bubble temperature).

There are two reasons why this underprediction of temperature gradient does not significantly impact the overall results of the thermal model. Firstly, the time of bubble collapse when these large gradients occur is extremely short compared to the 


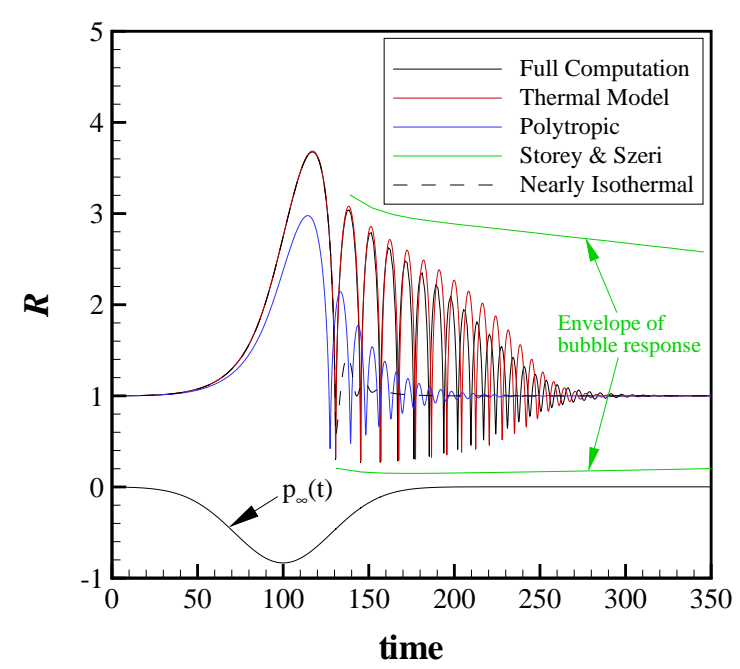

Figure 5. Computed bubble radius for an air bubble of equilibrium radius $R_{0}^{\prime}=40 \mu m(D=0.0287)$ in water subject to a Gaussian decrease in far field pressure $\left(A=1.0, t_{w}=40.0\right)$. Ambient conditions are $20^{\circ} \mathrm{C}$ and 1 atmosphere. The curves show comparisons to the full computation of the thermal model $(\alpha=7.524)$, the polytropic model with effective damping $\left(\beta=19.91, k_{\text {eff }}=1.213\right)$, the switching model of Storey \& Szeri (2001), and the nearly isothermal model of Prosperetti et al. (1991).
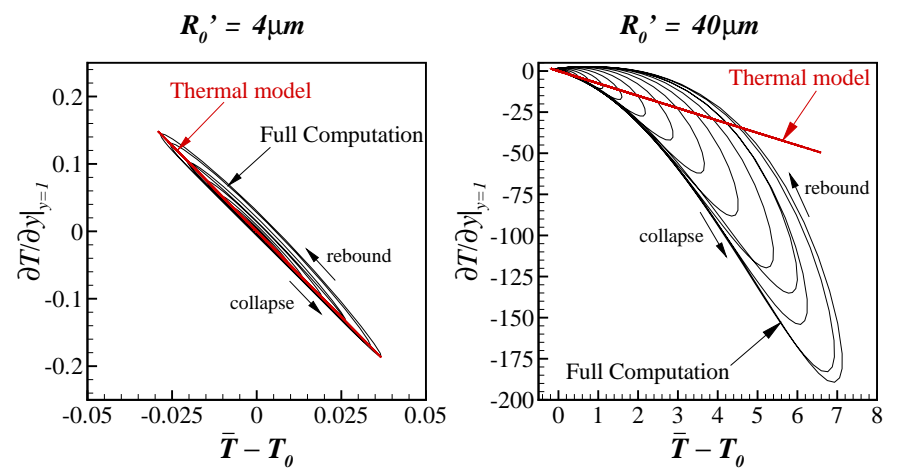

Figure 6. Temperature gradient at bubble wall as a function of average bubble temperature for full computation and thermal model for computations in Fig. $4\left(R_{0}^{\prime}=4 \mu \mathrm{m}\right)$ and Fig. $5\left(R_{0}^{\prime}=40 \mu \mathrm{m}\right)$.

overall period of a rebound cycle so that the model is only inaccurate for a very short period of time. Secondly, when large temperature gradients occur, the bubble is near its minimum radius so that the total surface area through which thermal energy is transferred by conduction is very small. Consequently this leads to only a slight underprediction of thermal energy loss, and hence only a slight underprediction of attenuation of the bubble rebounds.

We also compared the thermal model to the full computation for a $40 \mu m$ bubble subject to Gaussian pressure decreases with several different amplitudes and durations and found good agreement in all cases.

\section{EXTENSION TO INCLUDE MASS TRANSFER}

The simplified mass transfer model is an extension of the thermal model. Assuming that the ratios of specific heats of the non-condensible gas and vapor are equal Eq. (1) can be rederived to yield the following (Ichihara et al. 2000),

$$
\frac{d p}{d t}=\frac{3 \gamma}{R}\left(\left.\frac{p_{0} D}{R} \frac{\partial T}{\partial y}\right|_{y=1}-p \frac{d R}{d t}+\mathcal{R}_{v} T_{w} \dot{m}_{v}^{\prime \prime}\right) .
$$

Here $\mathcal{R}_{v}$ is the gas constant of the vapor and $\dot{m}_{v}^{\prime \prime}$ is the mass flux of vapor per unit area into the bubble. The last term represents an additional energy flux due to flux of vapor into or out of the bubble. To close the equations we need to be able to determine the value of $\dot{m}_{v}^{\prime \prime}$. From reciprocal diffusion, we can also write,

$$
\dot{m}_{v}^{\prime \prime}=\left.\rho \mathcal{D} \frac{1}{R} \frac{\partial C}{\partial y}\right|_{y=1},
$$

where $\rho, \mathcal{D}$ and $C$ are the gas mixture density, mass diffusivity of the gas/vapor mixture and mass concentration of vapor, all evaluated at the bubble wall. To avoid solving the full mass diffusion equations in the bubble we make a similar approximation to Eq. (6),

$$
\left.\frac{\partial C}{\partial y}\right|_{y=1} \approx-\alpha_{C}\left(\bar{C}-C_{w}\right)
$$

Here $C_{w}$ is the concentration of vapor at the wall which is determined by the assumption that the partial pressure of vapor is equal to the saturated vapor pressure. The volume averaged concentration, $\bar{C}$, is approximated by,

$$
\bar{C} \approx \frac{m_{v}}{m_{a_{0}}+m_{v}},
$$

where $m_{a_{0}}$ is the constant mass of non-condensible gas in the bubble and $m_{v}$ is the total mass of vapor in the bubble which is found by integrating $4 \pi R^{2} \dot{m}_{v}^{\prime \prime}$ in time. The value of $\alpha_{C}$ in Eq. (13) is again determined by matching to linear theory, but because the thermal and mass diffusivities for air/vapor mixtures are approximately the same, we simply choose $\alpha_{C}=\alpha$ for all the computations. Equations (11) through (14) constitute the new set of equations for the present model. The form of the Eq. (8) also has to be modified to allow for a non constant mass of bubble contents. The additional computational expense of the mass transfer model over the thermal model is only the integration of one extra ODE to determine the total mass of vapor in the bubble.

Figure 7 compares the bubble radius from a full computation $^{3}$ to the present mass transfer model. In addition we consider

\footnotetext{
${ }^{3}$ The full computation solves the energy and mass diffusion equations for the bubble interior using the Chebychev spectral collocation method.
} 


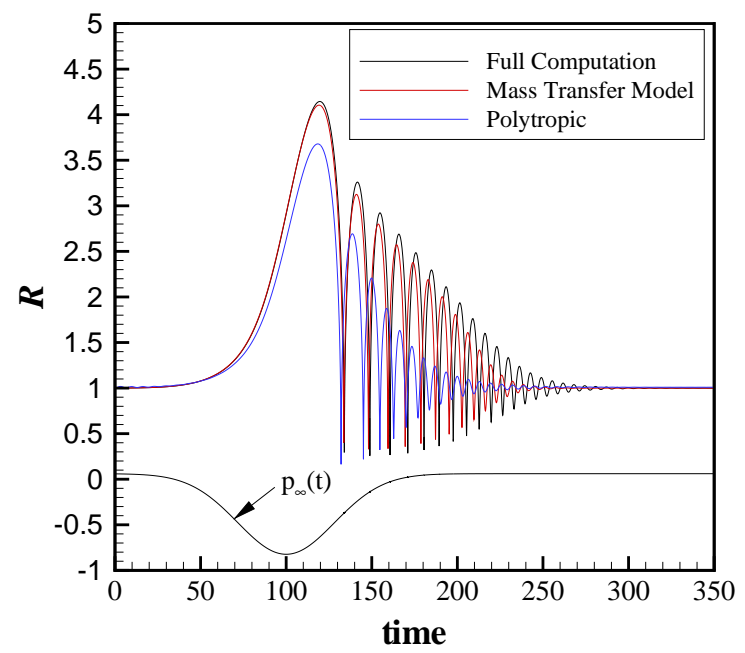

Figure 7. Computed bubble radius for an air/vapor bubble of equilibrium radius $R_{0}^{\prime}=40 \mu m(D=0.0284)$ in water subject to a Gaussian decrease in far field pressure $\left(A=1.0, t_{w}=40.0\right)$. Ambient conditions are $20^{\circ} \mathrm{C}$ and 1 atmosphere. The curves show comparisons to the full computation of the present model with $\alpha$ chosen to match linearized full equations $(\alpha=7.5)$ and the polytropic model with effective damping $\left(\beta=19.91, k_{\text {eff }}=1.213\right)$.

a polytropic model that assumes a constant vapor pressure. $\mathrm{Pa}$ rameters for the computations are the same as for Fig. 5 except for the addition of vapor. We see that the mass transfer model yields results similar to the thermal model, although rather than underpredicting the attenuation it overpredicts it, and rather than overestimating the period of bubble rebounds it slightly underpredicts it. The mass transfer model is superior to the polytropic model which suffers from the same problem as the case without vapor in that the initial expansion is grossly underpredicted.

\section{CONCLUSION}

A simple and efficient model that accounts for thermal damping effects in gas bubbles has been presented. The thermal model has been shown to have excellent agreement with expensive full bubble computations over a wide range of harmonic forcing frequencies and amplitudes. In addition, when subjected to more complex Gaussian forcings, the thermal model yields results that agree to full thermal computations much more closely than previous simple models. Near the isothermal limit the thermal model is equivalent to the nearly isothermal model of Prosperetti (1991), but away from this limit the thermal model does better than the nearly isothermal model.

The thermal model has also been extended to include mass diffusion of vapor within the gas and preliminary results indicate that this mass transfer model obtains results that agree much more closely with a full gas/vapor bubble computation than the polytropic model with effective damping and assumed constant vapor pressure. It now remains to incorporate the simple model into the bubbly continuum model to gauge what effects the diffusive damping has in a variety of bubbly cavitating flows.

\section{REFERENCES}

Colonius, T., d'Auria, F. \& Brennen, C. E. (2000), 'Acoustic saturation in bubbly cavitating flow adjacent to an oscillating wall', Physics of Fluids 12(11), 2752-2761.

Hao, Y. \& Prosperetti, A. (1999), 'The dynamics of vapor bubbles in acoustic pressure fields', Physics of Fluids 11(8), 20082019.

Ichihara, M., Kawashima, H. \& Kameda, M. (2000), 'Dynamics of a spherical gas/vapor bubble', Proceedings of the Third Annual Meeting of the Institute for Multifluid Science and Technology (IMuST).

Kamath, V. \& Prosperetti, A. (1989), 'Numerical integration methods in gas-bubble dynamics', J. Acoust. Soc. Am 85(4), 1538-1548.

Kameda, M. \& Matsumoto, Y. (1996), 'Shock waves in a liquid containing small gas bubbles', Physics of Fluids 8(2), 322-355. Lertnuwat, B., Sugiyama, K. \& Matsumoto, Y. (2001), 'Modeling of thermal behavior inside a bubble', Fourth International Symposium on Cavitation.

Preston, A. T., Colonius, T. \& Brennen, C. E. (2002), 'A numerical investigation of unsteady bubbly cavitating nozzle flows', Physics of Fluids 14(1), 300-311.

Prosperetti, A. (1991), 'The thermal behaviour of oscillating gas bubbles', Journal of Fluid Mechanics 222, 587-616.

Prosperetti, A., Crum, L. A. \& Commander, K. W. (1988), 'Nonlinear bubble dynamics', J. Acoust. Soc. Am. 83(2), 502-514.

Shimada, M., Kobayashi, T. \& Matsumoto, Y. (1999), 'Dynamics of cloud cavitation and cavitation erosion', Proceedings of the 1999 ASME/JSME Fluids Engineering Division Summer Meeting.

Storey, B. D. \& Szeri, A. J. (2001), 'A reduced model of cavitation physics for use in sonochemistry', Proc. R. Soc. Lond. A 457, 1685-1700.

van Wijngaarden, L. (1968), 'On the equations of motion for mixtures of liquid and gas bubles', Journal of Fluid Mechanics 33, 465-474.

van Wijngaarden, L. (1972), 'One-dimensional flow of liquids containing small gas bubbles', Annual Review of Fluid Mechanics 4, 369-396.

Wang, Y.-C. (1999), Stability analysis of one-dimensional steady cavitating nozzle flows with bubble size distribution, 1999 ASME/JSME FED Summer Meeting. 